\title{
A Note on the Men'shov-Rademacher Inequality
}

by

\section{Witold BEDNORZ}

\section{Presented by Stanistaw KWAPIEŃ}

Summary. We improve the constants in the Men'shov-Rademacher inequality by showing that for $n \geq 64$,

$$
\mathbf{E}\left(\sup _{1 \leq k \leq n}\left|\sum_{i=1}^{k} X_{i}\right|^{2}\right) \leq 0.11\left(6.20+\log _{2} n\right)^{2}
$$

for all orthogonal random variables $X_{1}, \ldots, X_{n}$ such that $\sum_{k=1}^{n} \mathbf{E}\left|X_{k}\right|^{2}=1$.

1. Introduction. We consider real or complex orthogonal random variables $X_{1}, \ldots, X_{n}$, i.e.

$$
\mathbf{E}\left|X_{i}\right|^{2}<\infty, \quad 1 \leq i \leq n, \quad \text { and } \quad \mathbf{E}\left(X_{i} X_{j}\right)=0, \quad 1 \leq i, j \leq n .
$$

Set $S_{j}:=X_{1}+\cdots+X_{j}$ for $1 \leq j \leq n$, and $S_{0}=0$. Clearly

$$
\mathbf{E}\left|S_{j}-S_{i}\right|^{2}=\sum_{k=i+1}^{j} \mathbf{E}\left|X_{k}\right|^{2} \quad \text { for } i \leq j .
$$

The best constant in the Men'shov-Rademacher inequality is defined by

$$
D_{n}:=\sup \mathbf{E} \sup _{1 \leq i \leq n}\left|S_{i}\right|^{2}
$$

where the supremum is taken over all orthogonal systems $X_{1}, \ldots, X_{n}$ which satisfy $\sum_{k=1}^{n} \mathbf{E}\left|X_{k}\right|^{2}=1$. We also define

$$
C:=\limsup _{n \rightarrow \infty} \frac{D_{n}}{\log _{2}^{2} n} .
$$

2000 Mathematics Subject Classification: Primary 26D15; Secondary 60E15.

Key words and phrases: inequalities, orthogonal systems.

Research supported by Grant P03A 01229 of Ministry of Science, Poland. 
Rademacher [6] in 1922 and independently Men'shov [5] in 1923 proved that there exists $K>0$ such that for $n \geq 2$,

$$
D_{n} \leq K \log _{2}^{2} n, \text { hence } C \leq K .
$$

By now there are several different proofs of the above inequality. The traditional proof of the Men'shov-Rademacher inequality uses the bisection method (see Doob [1] and Loève [4]), which leads to

$$
D_{n} \leq\left(2+\log _{2} n\right)^{2}, \quad n \geq 2, \quad \text { hence } \quad C \leq 1 .
$$

In 1970 Kounias [3] used a trisection method to get a finer inequality

$$
D_{n} \leq\left(\frac{\log _{2} n}{\log _{2} 3}+2\right)^{2}, \quad n \geq 2, \quad \text { hence } \quad C \leq\left(\frac{\log _{2} 2}{\log _{2} 3}\right)^{2} .
$$

S. Chobanyan, S. Levental and H. Salehi [2] proved the following result:

$$
D_{2 n} \leq \frac{4}{3} D_{n} \quad \text { if } D_{n} \leq 3 ; \quad D_{2 n} \leq\left(\left(D_{n}-\frac{3}{4}\right)^{1 / 2}+\frac{1}{2}\right)^{2},
$$

and as a consequence they got the estimate $D_{n} \leq \frac{1}{4}\left(3+\log _{2}^{2} n\right), C \leq \frac{1}{4}$. An example given in [2] shows that $D \geq \frac{\log _{2}^{2} n}{\pi^{2} \log _{2}^{2} e}$ and thus $C \geq 0.0487$. The aim of this paper is to improve the bisection method and together with (1) show that $C \leq 0.1107<\frac{1}{9}$.

\section{Results}

Theorem 1. For each $n, m \in \mathbb{N}$ and $l>2$,

$$
\sqrt{D_{n(2 m+l)}} \leq \sqrt{D_{n}}+\sqrt{\max \left\{D_{m}, 2 D_{l-1}\right\}}
$$

If $l=2$ then an even stronger inequality holds:

$$
\sqrt{D_{n(2 m+l)}} \leq \sqrt{D_{n}}+\sqrt{D_{m}}
$$

Proof. Set $p:=2 m+l$. We can assume that $\mathbf{E}\left|S_{p n}\right|^{2}=1$. The triangle inequality yields

$$
\left|S_{i}\right| \leq\left|S_{i}-S_{p j}\right|+\left|S_{p j}\right|
$$

Consequently,

$$
\max _{1 \leq i \leq p n}\left|S_{i}\right| \leq \max _{1 \leq i \leq p n} \min _{0 \leq j \leq n}\left|S_{i}-S_{p j}\right|+\max _{0 \leq j \leq n}\left|S_{p j}\right| .
$$

Thus

$$
\mathbf{E} \max _{1 \leq i \leq p n}\left|S_{i}\right|^{2} \leq \mathbf{E}\left(\max _{1 \leq i \leq p n} \min _{0 \leq j \leq n}\left|S_{i}-S_{p j}\right|+\max _{0 \leq j \leq n}\left|S_{p j}\right|\right)^{2} .
$$

The definition of $D_{n}$ together with the classical norm inequality implies

$$
\sqrt{D_{p n}} \leq \sqrt{D_{n}}+\sqrt{\mathbf{E} \max _{1 \leq i \leq p n} \min _{0 \leq j \leq n}\left|S_{i}-S_{p j}\right|^{2}} .
$$


It remains to show that

$$
\mathbf{E} \max _{1 \leq i \leq p n} \min _{0 \leq j \leq n}\left|S_{i}-S_{p j}\right|^{2} \leq \begin{cases}\max \left\{D_{m}, 2 D_{l-1}\right\} & \text { if } l>2 \\ D_{m} & \text { if } l=2 .\end{cases}
$$

Define

$$
\begin{aligned}
& A_{j}:=\max \left\{\left|S_{i}-S_{p j}\right|: p j \leq i \leq p j+m\right\}, \\
& B_{j}:=\max \left\{\left|S_{p(j+1)}-S_{i}\right|: p j+m+l \leq i \leq p(j+1)\right\}, \\
& C_{j}:=\max \left\{\left|S_{i}-S_{p j+m}\right|: p j+m<i<p j+m+l\right\}, \\
& D_{j}:=\max \left\{\left|S_{p j+m+l}-S_{i}\right|: p j+m<i<p j+m+l\right\},
\end{aligned}
$$

for each $j \in\{0,1, \ldots, n-1\}$. Each $0 \leq i \leq p n$ can be written in the form $i=p j+r$, where $j \in\{0, \ldots, n-1\}, r \in\{1, \ldots, p\}$. If $r \leq m$, then

$$
\left|S_{i}-S_{p j}\right|^{2} \leq A_{j}^{2}
$$

If $r \geq m+l$, then

$$
\left|S_{p(j+1)}-S_{i}\right|^{2} \leq B_{j}^{2}
$$

The last case is when $i=p j+m+r, r \in\{1, \ldots, l-1\}$. Set

$$
\begin{array}{rlrl}
P_{j} & :=S_{p j+m}-S_{p j}, & V_{j} & :=S_{p j+m+r}-S_{p j+m}, \\
Q_{j} & :=S_{p(j+1)}-S_{p j+m+l}, & W_{j}:=S_{p j+m+l}-S_{p j+m+r} .
\end{array}
$$

Clearly $(i=p j+m+r, r \in\{1, \ldots, l-1\})$

$$
\min \left\{\left|S_{i}-S_{p j}\right|^{2},\left|S_{p(j+1)}-S_{i}\right|^{2}\right\}=\min \left\{\left|P_{j}+V_{j}\right|^{2},\left|Q_{j}+W_{j}\right|^{2}\right\} .
$$

For all complex numbers $a, b, c, d$ we have

$$
\frac{1}{2}|a+b|^{2} \leq|a|^{2}+|b|^{2}, \quad \frac{1}{2}|c+d|^{2} \leq|c|^{2}+|d|^{2} .
$$

Since

$$
\min \left\{|a+b|^{2},|c+d|^{2}\right\} \leq \frac{1}{2}|a+b|^{2}+\frac{1}{2}|c+d|^{2}
$$

we obtain

$$
\min \left\{|a+b|^{2},|c+d|^{2}\right\} \leq|a|^{2}+|b|^{2}+|c|^{2}+|d|^{2}
$$

Hence

$$
\min \left\{\left|S_{i}-S_{p j}\right|^{2},\left|S_{p(j+1)}-S_{i}\right|^{2}\right\} \leq\left|P_{j}\right|^{2}+\left|Q_{j}\right|^{2}+\left|V_{j}\right|^{2}+\left|W_{j}\right|^{2},
$$

and consequently for each $p j<i \leq p(j+1), j \in\{0,1, \ldots, n-1\}$,

$$
\min \left\{\left|S_{i}-S_{p j}\right|^{2},\left|S_{p(j+1)}-S_{i}\right|^{2}\right\} \leq A_{j}^{2}+B_{j}^{2}+C_{j}^{2}+D_{j}^{2} .
$$

In fact we have proved that

$$
\mathbf{E} \max _{1 \leq i \leq p n} \min _{0 \leq j \leq n}\left|S_{i}-S_{p j}\right|^{2} \leq \mathbf{E} \sum_{j=0}^{n-1}\left(A_{j}^{2}+B_{j}^{2}+C_{j}^{2}+D_{j}^{2}\right) .
$$


Observe that

$$
\begin{aligned}
& \mathbf{E} A_{j}^{2} \leq D_{m} \sum_{k=1}^{m} \mathbf{E}\left|X_{p j+k}\right|^{2}, \quad \mathbf{E} B_{j}^{2} \leq D_{m} \sum_{k=1}^{m} \mathbf{E}\left|X_{p j+m+l+k}\right|^{2}, \\
& \mathbf{E}\left(C_{j}^{2}+D_{j}^{2}\right) \leq D_{l-1}\left(\mathbf{E}\left|X_{p j+m+1}\right|^{2}+\mathbf{E}\left|X_{p j+m+l}\right|^{2}+2 \sum_{k=2}^{l-1} \mathbf{E}\left|X_{p j+m+k}\right|^{2}\right),
\end{aligned}
$$

Notice that if $l=2$ then

$$
\mathbf{E}\left(C_{j}^{2}+D_{j}^{2}\right) \leq D_{1}\left(\mathbf{E}\left|X_{p j+m+1}\right|^{2}+\mathbf{E}\left|X_{p j+m+1}\right|^{2}\right) .
$$

Hence, if $l>2$ then

$$
\mathbf{E} \max _{1 \leq i \leq p n} \min _{0 \leq j \leq n}\left|S_{i}-S_{p j}\right|^{2} \leq \max \left\{D_{m}, 2 D_{l-1}\right\}
$$

and if $l=2$ then

$$
\mathbf{E} \max _{1 \leq i \leq p n} \min _{0 \leq j \leq n}\left|S_{i}-S_{p j}\right|^{2} \leq D_{m}
$$

This ends the proof.

Corollary 1. For each $n \geq m$,

$$
D_{n} \leq D_{m}\left(2+\frac{\log _{2} n-\log _{2} m}{\log _{2}(2 m+2)}\right)^{2} .
$$

Proof. Taking $l=2$ in Theorem 1 we obtain

$$
D_{m(2 m+l)^{k}} \leq(k+1)^{2} D_{m}
$$

For each $n \geq m$ there exists $k \geq 0$ such that $m(2 m+l)^{k} \leq n<m(2 m+l)^{k+1}$. Hence

$$
k \leq 1+\frac{\log _{2} n-\log _{2} m}{\log _{2}(2 m+2)} .
$$

Consequently,

$$
D_{n} \leq D_{m}\left(2+\frac{\log _{2} n-\log _{2} m}{\log _{2}(2 m+2)}\right)^{2}
$$

This result implies

$$
C=\limsup _{n \rightarrow \infty} \frac{D_{n}}{\log _{2}^{2} n} \leq \frac{D_{m}}{\log _{2}^{2}(2 m+2)} .
$$

Putting $l>2$ in Theorem 1 and proceeding as in the proof of Corollary 1 we get the following result.

Corollary 2. For each $l>2$ and $n \geq m$,

$$
D_{n} \leq \max \left\{D_{m}, 2 D_{l-1}\right\}\left(2+\frac{\log _{2} n-\log _{2} m}{\log _{2}(2 m+l)}\right)^{2} \text {. }
$$

Consequently,

$$
C \leq \frac{\max \left\{D_{m}, 2 D_{l-1}\right\}}{\log _{2}^{2}(2 m+l)}
$$


As mentioned in the introduction, $D_{2}=4 / 3$ (by the result of Chobanyan, Levental, and Salehi [2]). Hence applying Corollary 1 with $m=2$ we get

$$
D_{n} \leq \frac{4}{3 \log _{2}^{2} 6}\left(2 \log _{2} 6-\log _{2} n\right)^{2}, \quad \text { and } \quad C \leq \frac{4}{3 \log _{2}^{2} 6}<\frac{1}{5} .
$$

It follows by (1) that

and

$$
D_{2}=\frac{4}{3}, \quad D_{4} \leq\left(\frac{4}{3}\right)^{2}, \quad D_{8} \leq\left(\frac{4}{3}\right)^{3}, \quad D_{16} \leq\left(\frac{4}{3}\right)^{4}
$$

$$
D_{32} \leq\left(\left(D_{16}-\frac{3}{4}\right)^{1 / 2}+\frac{1}{2}\right)^{2}, \quad D_{64} \leq\left(\left(D_{32}-\frac{3}{4}\right)^{1 / 2}+\frac{1}{2}\right)^{2} .
$$

Hence

$$
D_{8} \leq 2.3704, \quad D_{64} \leq 5.5741 .
$$

Taking $m=64, l=9$ we get

$$
\frac{\max \left\{D_{m}, 2 D_{l-1}\right\}}{\log _{2}^{2}(2 m+l)} \leq 0.1107<1 / 9 .
$$

Thus applying Corollary 2 (with $m=64, l=9$ ) we find that for each $n \geq 64$,

$$
D_{n} \leq 0.1107\left(2 \log _{2}(137)-8+\log _{2} n\right)^{2} \leq 0.1107\left(6.1960+\log _{2} n\right)^{2} .
$$

This gives the estimate $C \leq 0.1107<\frac{1}{9}$.

\section{References}

[1] J. L. Doob, Stochastic Processes, Wiley, New York, 1953.

[2] S. Chobanyan, S. Levental and H. Salehi, On the best constant in the RademacherMenchov inequality, J. Inequal. Appl., to appear.

[3] E. G. Kounias, A note on Rademacher's inequality, Acta Math. Acad. Sci. Hungar. 21 (1970), 447-448.

[4] M. Loève, Probability Theory, D. Van Nostrand, 1960.

[5] D. Menchoff [D. Men'shov], Sur les séries de fonctions orthogonales, Fund. Math. 4 (1923), 82-105.

[6] H. Rademacher, Einige Sätze über Reihen von allgemeinen Orthogonalfunktionen, Math. Ann. 87 (1922), 112-138.

Witold Bednorz

Institute of Mathematics

Warsaw University

Banacha 2

02-097 Warszawa, Poland

E-mail: wbednorz@mimuw.edu.pl

Received January 19, 2006;

received in final form May 18, 2006 